\title{
Présentations et représentations des nouvelles esthétiques de la céramique
}

\section{Elisa Ullauri Lloré}

\section{(2) OpenEdition \\ Journals}

Édition électronique

URL : https://journals.openedition.org/tc/7593

DOI : $10.4000 /$ tc. 7593

ISBN : 0248-6016

ISSN : 1952-420X

Éditeur

Éditions de l'EHESS

\section{Édition imprimée}

Date de publication : 24 décembre 2015

Pagination : 230

ISBN : 978-2-7132-2505-5

ISSN : 0248-6016

\section{Référence électronique}

Elisa Ullauri Lloré, «Présentations et représentations des nouvelles esthétiques de la céramique », Techniques \& Culture [En ligne], 64 | 2015, mis en ligne le 24 mars 2016, consulté le 29 septembre 2022. URL : http://journals.openedition.org/tc/7593 ; DOI : https://doi.org/10.4000/tc.7593

Ce document a été généré automatiquement le 29 septembre 2022.

Tous droits réservés 


\title{
Présentations et représentations des nouvelles esthétiques de la céramique
}

\author{
Elisa Ullauri Lloré
}

\section{NOTE DE L'ÉDITEUR}

Nous vous invitons à consulter la synthèse de cet article (édition papier) en téléchargeant le document placé en annexe.

$$
\begin{array}{r}
\text { «Il y a ce qu'il faut détruire, et il y a ce qu'il faut } \\
\text { simplement éclairer et regarder. L'examen } \\
\text { bienveillant est grave, quelle force ! N'apportons } \\
\text { point la flamme là où la lumière suffit ». } \\
\text { Victor Hugo, Les Misérables }
\end{array}
$$

"Cette pièce est une "cristallisation" sur porcelaine, elle a été cuite à $1280^{\circ} \mathrm{C}$. C'est une technique vraiment complexe et capricieuse... mais infinie. En fait, dans mon travail la technique accompagne l'objet et non l'inverse ».

1 Cette phrase a été prononcée par Stéphan, un céramiste présent à la Biennale internationale de la céramique, Argilla, Aubagne, en 2013. D'un autre côté, Michelle et Xavier, un couple de collectionneurs de céramique expriment leur attachement à la dimension technique de la céramique :

«La technique c'est ce qui nous intéresse. C'est d'ailleurs pour ça qu'on assiste aux cuissons, on visite les ateliers... quand on va dans les galeries parfois on se dit : mais pourquoi vous n'expliquez pas aux gens comment s'est fait? Quand on pense à Girel par exemple, son truc là passe 7 ou 8 fois au four... ça veut dire qu'il y a du temps, une connaissance folle, il y a du gaz et un tas de trucs que justifient les prix des choses (...) Il faut leur expliquer... dites aux gens comment s'est fait !».

2 À l'image de ces énoncés, les discours émis sur la céramique d'art sont fréquemment teintés par une rhétorique de la technique. Au sein du système de médiation, c'est la 
technique qui est invoquée pour décrire et évaluer les objets, même si, comme Stephan, certains préfèrent la considérer comme secondaire. La prévalence de la notion de la technique dans les discours entourant les objets et les pratiques de la céramique renvoie à la construction d'un secteur artistique ancré dans un régime ambivalent entre «l'art et le métier» (Bajard 2012, Jourdain 2012) mais dont se détachent de plus en plus de nouvelles conceptions. Le présent article propose d'interroger la manière dont la technique permet d'articuler les discours sur la céramique. En quoi est-elle déterminante pour la construction ambivalente de la céramique? Selon quelles logiques se construisent les représentations et les imaginaires de la céramique? Dans quelles dynamiques sociales et artistiques s'inscrit-elle?

3 Dans la définition générale de la céramique se trouve le processus par lequel l'objet fait d'argile subit des transformations chimiques sous l'action du feu (à des températures supérieures à $700^{\circ} \mathrm{C}$ ). Son acception la plus courante illustre d'un côté l'hétérogénéité des matières ${ }^{1}$ et de l'autre, la spécificité de l'objet fini, après cuisson:

« l'art de façonner l'argile et d'en fixer les formes par la cuisson. Le mot céramique désigne des produits de composition et d'apparence diverses, ayant pour base l'argile ou toute terre plastique » (Brunet et al. 2015).

Du service en porcelaine de la grand-mère aux vestiges archéologiques issus de cultures anciennes, ou de l'application industrielle des matériaux céramiques à l'expression artistique, le terme "céramique " revêt une multiplicité d'objets, de pratiques et de représentations de différentes natures. Elle parle divers langages; que ce soit la sculpture, le design, l'artisanat d'art ; et son vocabulaire c'est sa matérialité. Faire de la céramique nécessite donc une instruction complexe comprenant non seulement la connaissance des matières, des techniques, des formules chimiques des émaux, des terres et argiles, mais aussi des connaissances liées à l'usage d'instruments et équipements divers tels que le four, le tour, etc. 
Figure 1

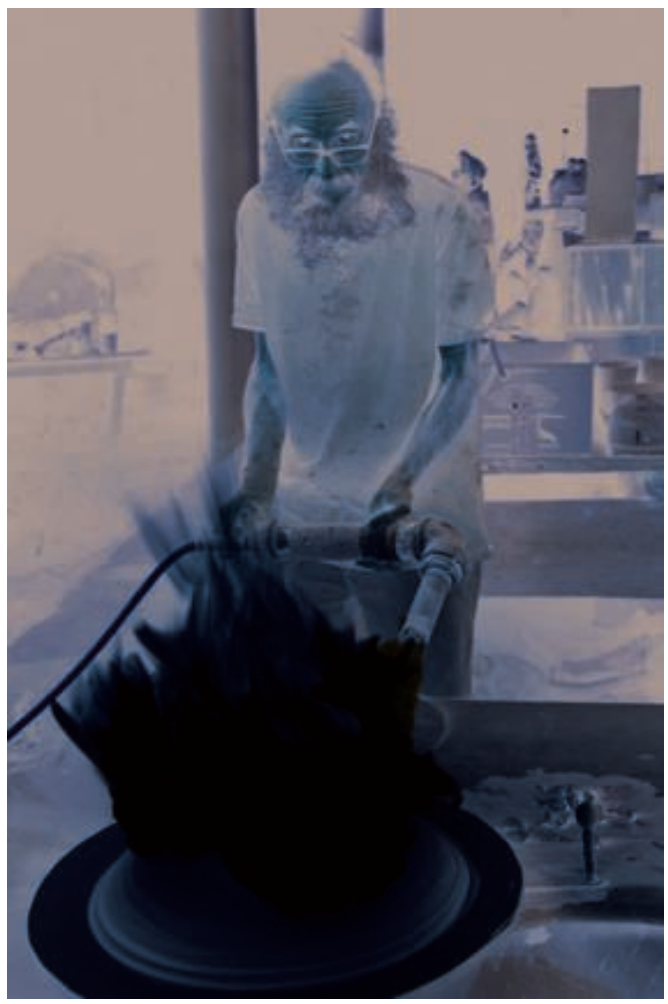

Céramiste en train de sécher une pièce en grés tournée à feu direct

(cl. Angela Jiménez, 2014)

5 Le propos de cet article est d'interroger l'articulation des discours et des positionnements des amateurs et des spécialistes de la céramique sur l'état actuel de cet espace artistique. Il ne s'agit point de faire une étude esthétique des objets, mais d'interroger la place et le rôle qu'ont les acteurs spécialistes et amateurs de céramique dans la reconfiguration des représentations du milieu, les changements socioesthétiques et la redéfinition des frontières esthético-sociales. À travers le témoignage de céramistes, amateurs, collectionneurs, intermédiaires, etc., l'objectif est de saisir les mécanismes du processus de transformation de l'opérativité symbolique, sociale, matérielle, de la céramique. Dans ces processus, nous verrons comment les questions liées à la technique contribuent à la construction d'un système spécifique, dont les normes et discours se transforment notamment par le contact avec l'art contemporain.

\section{Questions de méthode}

Les acteurs étudiés témoignent de nombreux engagements communs et différentiels à la fois. En adossant les discours et récits des acteurs sociaux et institutionnels du monde de la céramique aux interrogations sur la technique, l'analyse découle des résultats d'une enquête ethnosociologique dans le cadre de ma thèse de doctorat. Les outils méthodologiques mobilisés ont été le questionnaire, l'entretien semi-directif et un travail d'observation des activités proposées par le Club de Collectionneurs de Céramique pour et par ses membres (conférences, visites d'expositions, salons, vernissages, collections privées). Ce lieu fédérateur d'amateurs ${ }^{2}$ de céramique a été créé en 2012 et compte environ 150 membres. Il est constitué non seulement par des 
collectionneurs, mais aussi par des galeristes, conservateurs, professionnels, etc. L'étude se veut

«au plus près des situations naturelles des sujets (...) afin de produire des connaissances in situ, contextualisées, transversales, visant à rendre compte du "point de vue" de l'acteur » (Olivier de Sardan 1995).

Son ancrage géographique est majoritairement celui du paysage artistique français. Toutefois, certains enquêtés sont originaires d'autres pays, principalement d'Europe, mais ont pour point commun d'entretenir un lien avec la scène céramique française. Cette condition s'appuie sur l'hypothèse qu'il existe dans le milieu un fort sentiment d'appartenance à une communauté artistique autonome, s'étendant au-delà des frontières et dont le langage est compris par ses membres malgré la pluralité de branches et ramifications qui se croisent.

\section{Une nouvelle scène artistique polarisée}

La céramique se structure selon une double appartenance tantôt revendiquée, tantôt contestée aux logiques de l'artisanat d'art et de l'art (et dans une rupture ou une continuité avec une culture céramique). L'évolution de la céramique actuelle s'accompagne d'une série de mutations produites chez les créateurs et les œuvres, les espaces de création, d'exposition, de diffusion et de médiation visibles dans l'interaction de matières, de techniques et de supports, ainsi que dans le renouveau des imaginaires qui lui sont étroitement associés.

\section{La céramique d'art et sa pratique majoritaire}

D'un côté, les pratiques majoritaires de la céramique d'art empruntent les compétences, les normes et les éthiques du régime artisanal tout en s'exerçant en «régime de singularité » artistique (Heinich 2005). La production se retrouve principalement dans les marchés potiers, - espaces de commercialisation des pièces, de diffusion de la pratique ainsi que de sociabilité entre membres du groupe professionnel -, ainsi que dans des salons de métiers d'art. Serge Nicole, directeur d'Ateliers d'Art de France ${ }^{3}$ et céramiste, avançait lors d'une interview, que la céramique joue un rôle fondamental dans l'évolution de l'ensemble du secteur des métiers d'art :

«La céramique a un rôle de moteur de la structuration du secteur (des métiers d'art) et pour la dissolution des barrières entre l'artisanat et l'art contemporain. La céramique est motrice effectivement pour l'accession dans l'image du public; les galeries incorporent la céramique avant les autres métiers d'art et donc la céramique a ce rôle-là d'aider à la dissolution des frontières ».

Pour lui, même si la céramique a son identité culturelle forte, l'évolution doit se penser pour l'ensemble des métiers d'art à travers des partenariats stratégiques avec l'ensemble des acteurs:

«le fait d'avoir noué des partenariats stratégiques avec un ensemble d'acteurs constitue d'autant plus une communauté élargie, professionnelle, une communauté plus vaste d'acteurs qui sont engagés aussi dans la structuration: la maison des artistes, le ministère de la Culture, le ministère du Commerce et de l'Artisanat, mais aussi avec de plus petits groupes comme le club de collectionneurs de céramique... et c'est ça qui fait notre force aujourd'hui ».

Le projet de réconcilier l'art et l'artisanat reste néanmoins structurant en termes de positionnement des professionnels au sein du secteur (Jourdain 2012). Ainsi, cette catégorie regroupe les pratiques majoritaires de la céramique d'art à travers la formalisation des normes professionnelles dont Flora Bajard livre un portrait 
remarquable dans sa thèse. En effet, la génération qu'elle nomme les « fondateurs » du métier correspond à celle qui a fait émerger de nouvelles pratiques à partir des années 1940, «par des logiques successives de spécialisation, d'amalgame dans la division du travail et de transmutation de pratiques professionnelles antérieures» (Bajard 2014). Une seconde génération des «bâtisseurs " du métier de céramiste va apparaître dans les années 1970. Elle a « codifié et constitué un corpus de normes partagées » instituant un capital spécifique au groupe.

\section{La céramique contemporaine et ses pratiques minoritaires}

De ces pratiques majoritaires et instituées, se détachent d'autres plus récentes et dans une certaine marge. Celles-ci s'inscrivent dans un processus d'" artification " (Heinich \& Shapiro 2012) plus accomplie que les pratiques dominantes. Ces pratiques minoritaires participent du décloisonnement de la céramique contemporaine par leur transversalité plus au moins marquée avec l'art contemporain. Que ce soit dans les musées, les écoles d'art, les institutions muséales, les galeries ou encore chez les collectionneurs, on voit émerger de nouveaux discours sur le renouveau de la céramique. Cependant, qu'il s'agisse du contournement des gestes traditionnels ou d'une maitrise technique des plus remarquable, ces changements semblent encore peu explorés. Ces derniers entrants du monde de la céramique peuvent être rapprochés de ce que Howard Becker appelait les francs-tireurs ${ }^{4}$; en ce qu'ils apportent des innovations en transgressant les normes et conventions construites par les membres intégrés. Cette création céramique contemporaine se manifeste par un large éventail de propositions.

Figure 2

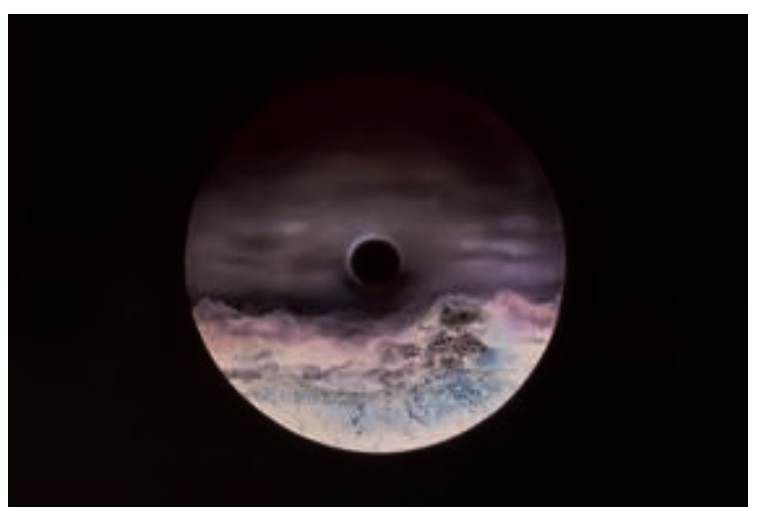

Jean Girel, Paysage Bourgogne, porcelaine, 2014. www.jeangirel.fr (cl. Jean Girel, 2014) 
Figure 3

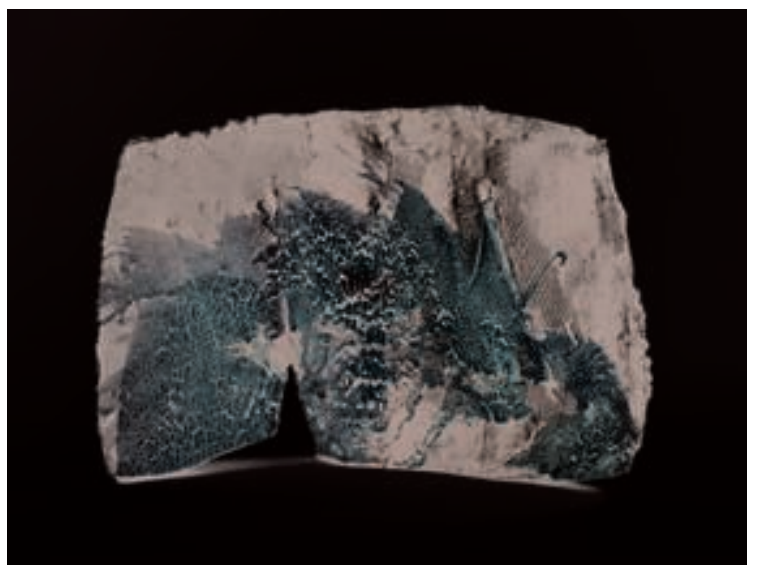

Claude Champy, Falaise, grès, 2019

(cl. Cécile Champy, 2015)

\section{Figure 4}

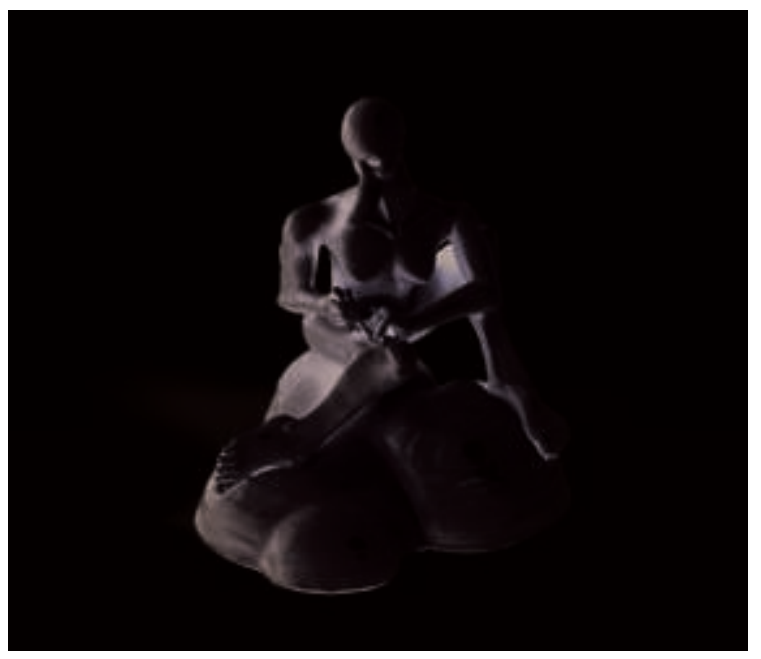

Louise Hindsgavl, Feeling love, 2006. www.louisehindsgavl.dk (cl. Courtoisie de l'artiste, 2014)

Figure 5

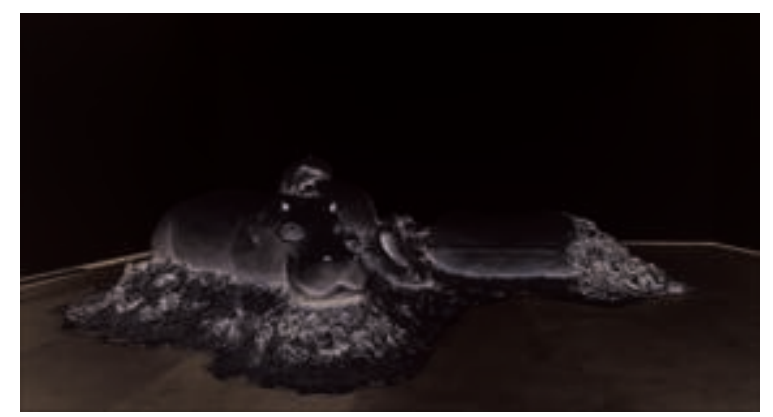

Daniel Dewar et Grégory Gicquel, Sans titre, kaolin, 2007. www.loevenbruck.com (cl. Galerie Loevenvruck, Paris, 2015) 
6 basculement axiologique de la pratique. Ces mutations sont visibles au prisme de nouvelles esthétiques, comme le montrent les œuvres de Jean Girel ou de Claude Champy à travers les traces d'une forte technicité articulée à l'innovation de la conception traditionnelle de la céramique. Certaines œuvres accordent également de nouvelles acceptions à la pratique. En déjouant la matérialité même des œuvres, elles proposent une réflexion sur les propriétés et les limites de la céramique. Le cas des hippopotames, une œuvre monumentale réalisée en terre crue par le duo d'artistes Dewar et Gicquel, et montrée lors de la Biennale internationale de la création contemporaine et céramique de Vallauris, en 2010, interroge : est-ce de la céramique malgré l'absence de cuisson? Aussi, l'œuvre de Louise Hindsgavl montre le contraste entre les représentations de la «belle» porcelaine blanche ${ }^{5}$ et le traitement contemporain de la matière. Cette pièce rappelle de loin les figurines décoratives en porcelaine par la couleur et la finesse de la manufacture, mais déroute de près par la violence de la scène et son sujet. Ces nouvelles esthétiques participent ainsi de la polarisation du milieu et redéfinissent les catégories historiquement bâties par et pour le milieu.

7 Le glissement du monde de la céramique vers les mondes de l'art contemporain s'est opéré dans l'espace professionnel et ses espaces de diffusion. Diverses manifestations et événements, dont on peut donner un bref historique des plus récents, ont été les vitrines de ces mutations. En 2003, Grayson Perry gagnait le Prix Tourner ${ }^{6}$ avec son travail pictural sur des vases en céramique. En 2005, la Nouvelle Biennale de céramique contemporaine de Châteauroux prenait un tout nouveau tournant ${ }^{7}$ en mettant à l'honneur la céramique dans l'art contemporain et les aspects les plus novateurs de la discipline. En 2010, les Ateliers d'Art de France avec les Arts décoratifs de Paris et la Cité de la céramique, Sèvres, en partenariat avec une quarantaine de galeries parisiennes et centres culturels à Paris, organisaient «Circuits Céramiques » à l'occasion de la $44^{\mathrm{e}}$ assemblée générale de l'Académie internationale de la Céramique. Avec de nombreuses expositions et rétrospectives, cette manifestation mettait l'accent sur la place grandissante de la céramique dans l'art contemporain et « le renouveau des pratiques autour d'un matériau qui a connu un indéniable regain d'intérêt dans le champ artistique tout entier ${ }^{8}$. En 2014 s'est tenue la XXIII ${ }^{\mathrm{e}}$ Biennale internationale de Vallauris, Création contemporaine et céramique, où «tous les deux ans, la ville de Vallauris se démultiplie pour valoriser toutes les facettes de la céramique internationale » [...] et où « le visiteur pourra apprécier [...] une démarche artistique singulière [...] dépassant la frontière traditionnelle entre l'objet fonctionnel et la pièce unique ». En 2016, Céramix, une exposition présentée à Maastricht (Bonnenfaten Museum), à Sèvres (Cité de la céramique) et à Paris (La Maison Rouge), réunit environ 250 œuvres de plus de cent artistes du monde entier et met en lumière l'intérêt historique des artistes pour la céramique. Il s'agit, selon les commissaires d'exposition Camille Morineau et Lucia Pesapane, de montrer une histoire de l'art parallèle et méconnue du grand public. Le projet de dépasser des frontières traditionnelles renvoie ainsi aux implications d'une partie de la création actuelle pour la reconnaissance artistique de la céramique et son décloisonnement. 


\section{Une rhétorique de la technique}

Comment peut-on donc saisir, mesurer et traduire ces changements ? Les présentations et représentations de la céramique artistique vues à travers le prisme de la technique permettent d'observer la construction des sens au cœur d'un espace artistique composite, en continuité ou en rupture avec les normes et pratiques d'une «culture artistique " attachée à sa dimension technique. La notion de technique permet de distinguer les subjectivités mobilisées dans les représentations des discours légitimant ou délégitimant certaines formes de création. La technique a été traitée en sciences humaines par de nombreux auteurs tels que Bruno Latour, Michel de Certeau, Alfred Gell, etc. Comprise comme un phénomène social, la technique est considérée par certains auteurs au-delà de la compétence et du savoir-faire, mais dans une chaîne de processus complexes visibles à travers les représentations que les acteurs ont des objets et des pratiques. Celle-ci présente des effets tels que la virtuosité, la performativité, la prouesse, la contorsion, etc. (Stoichita 2011). La céramique intègre ainsi un modèle dont la technique est un des fondamentaux. Cependant, son caractère incontournable devient dépassable et fréquemment réinterrogé. Alfred Gell oppose la conception purement utilitariste de la technique à la «caractéristique potentielle de toute action» :

«Ce qui distingue la "technique" de la non-technique est un certain degré de contournement dans l'obtention d'un résultat donné. [...] Les moyens techniques sont des moyens détournés d'atteindre un but. Le degré de technicité est proportionnel au nombre et à la complexité des étapes qui lient le donné initial à l'objectif poursuivi » (Gell $1988: 6$, trad. par Stoichita et al. 2011).

Selon la pensée de Gell, le contournement de la technique et la considération des procédés techniques ne signifient pas l'absence de technique, mais traduisent le déploiement d'un ensemble d'étapes venant déterminer le résultat final.

\section{La médiation par la technique}

Ce que nous désignons comme une "rhétorique de la technique» renvoie aux dispositifs de médiation de la céramique à travers la mise en scène d'une culture de métier et d'une identité professionnelle chez les céramistes d'art (Bajard 2014). Cette identité est exprimée par la connaissance (et la reconnaissance) des savoir-faire et des processus techniques partagés par les membres de la communauté artistique. Les situations de communication à travers lesquelles se produit la médiation de la céramique montrent comment se négocient les explications techniques entre "décontextualisation» et "réduction des contenus scientifiques». Les dispositifs explicatifs et interprétatifs de la médiation écrite introduisent le principe de la transmission des savoirs. Ce processus est visible à travers l'univers symbolique des expositions :

«L'exposition suppose un jeu social entre production et réception : une production

pour un public et une réception pour accéder à un monde » (Davallon 2000).

En effet, il n'est pas rare qu'en visitant une exposition de céramique, un musée ou un salon, les textes explicatifs et les différents dispositifs de la médiation mobilisent une rhétorique de la technique: de l'illustration des processus techniques des œuvres (cuisson, tournage, émaillage...) à la mise en scène de l'atelier du céramiste. Le travail de transmission et de traduction s'accompagne aussi par la mise en place d'ateliers pédagogiques, visites thématiques, stages, démonstrations techniques, etc. Anne 
Jourdain, note que la construction de la singularité des métiers d'art se trouve instituée à travers un système de règles définissant les frontières du marché :

«En valorisant la technicité, mais aussi l'aspect non standardisé de leur processus de fabrication, ils tentent de différencier leurs objets des produits industriels et des produits amateurs dont ils subissent la concurrence par les prix » (Jourdain 2012).

Le système mettant en avant le geste de la main, la technicité, le savoir-faire, etc., se trouve de plus en plus bouleversé par l'apparition de pratiques minoritaires, et se situe au croisement de la céramique d'art et l'art contemporain. Les stratégies communicationnelles valorisant la technicité sont perçues par certains acteurs comme étant indispensables, mais elles sont aussi décriées par d'autres du fait qu'elles pourraient se constituer comme un frein à l'accès et à la reconnaissance de la céramique par le monde de l'art. Frédéric Bodet, conservateur des collections contemporaines de la Cité de la céramique, Sèvres, considère par exemple :

« [qu'] on a trop tendance à parler technique quand on montre de la céramique ; d'essayer d'intéresser le public à la céramique par la technique. Car justement la céramique a été montrée dans des musées d'arts appliqués, d'arts décoratifs, d'artisanat d'art où la notion technique est primordiale. Si on veut que la céramique soit montrée dans un musée d'art contemporain, elle ne sera pas appréhendée par la technique (...) Là, on est dans un musée qui fait la part belle à une spécialité (musée de Sèvres), dans un domaine particulier, un musée lié aussi à une manufacture. Donc évidemment on parle technique ».

La volonté d'ouvrir la céramique à d'autres traitements discursifs et stratégies communicationnelles (à travers de nouveaux dispositifs de médiation), devrait s'effectuer, soutient Bodet, sur la base d'une négociation qui ne saurait négliger ni le discours axé sur la matière et ses processus techniques, ni celui porté sur les registres fondés sur l'expérience sensible. En ce sens, les subjectivités mobilisées dans les discours des amateurs de céramique, légitimant ou délégitimant certaines formes de création par rapport à d'autres, passent aussi par une opinion construite autour du trait de la technique. Dans le questionnaire proposé aux membres du club, trois réponses étaient possibles pour définir la place de la technique: fondamentale, secondaire et sans importance. Un peu plus de la moitié (soit $52 \%$ ) considère la technique comme un élément secondaire, quand $47 \%$ déclarent percevoir la technique comme étant fondamentale et qu'elle devrait être expliquée systématiquement. Seulement $1 \%$ répondra qu'elle n'a pas d'importance.

\section{Entre force et liberté dans la technique}

Cette considération de la technique nous permet maintenant de repenser les contours et les frontières des nouvelles esthétiques de la céramique contemporaine. Une distinction entre céramique à technique forte et céramique à technique libre a été saisie dans les discours des acteurs sur les objets et les pratiques. Le double traitement de la technique dans la céramique dessine ainsi une ligne entre deux conceptions de création. On distingue une polarisation à partir de l'usage et l'application de la technique en tant que système de légitimation et d'évaluation de la valeur des objets. Cependant, cette distinction admettrait un partage qui est nuancé par pléthore de réalités ${ }^{9}$. Il montre les différences intercatégorielles et les espaces intermédiaires, tout en tenant compte des complexités individuelles (Lahire 2012). Les exemples et postulats qui suivent se dégagent de cette distinction dont l'avantage est qu'elle permet de visualiser les contours flous à la base de nombreuses tensions dans le milieu. 


\section{Force et distance de la technique}

La notion de technique forte renvoie au groupe de céramistes artistes ayant recours au système de techniques et de normes de la "culture céramique ", tout en valorisant une certaine distance. Elle se trouve en continuité partielle (Davallon 2006) avec la «tradition céramique » puisqu'elle opère une remise à jour d'une pratique considérée comme peu visible dans la scène artistique. Cette catégorie pointe une forme de création caractérisée par la recherche d'une forte technicité et l'attachement à la culture du métier, mais revendique à la fois son appartenance à un univers artistique plus large. La valeur esthétique des objets est associée et validée par la maîtrise technique inhérente au processus de fabrication et au savoir-faire du créateur. Les traces d'une virtuosité technique sont visibles dans et par la matérialité de l'objet; de la manufacture de la pièce à la pose de l'émail, la cuisson de la pièce, la technique employée (raku, céladon, terre vernissée, porcelaine, cristallisation, etc.), mais leur valeur est associée à une liberté dans la création, gage de légitimité artistique dans le sens le plus noble de la pratique.

Des céramistes consacrés dans le monde de la céramique comme Claude Champy, Agathe Larpent-Ruffe, Jean Girel, Camille Virot, Bernard Dejonghe, etc. en constituent quelques exemples. Considérés " maitres » d'une céramique artistique et technique, ils sont généralement issus des écoles d'art, ont participé à la construction des réseaux du groupe professionnel (associations, organisations diverses, symposiums, maisons d'édition, etc.). Les céramistes de ce groupe sont pour certains membres de l'Académie internationale de la céramique (AIC) ${ }^{10}$, leurs œuvres font partie des collections publiques (musée de Sèvres, musées des Arts décoratifs de Paris, etc.) et privées. Leur consécration artistique est plus importante que celle de leurs pairs céramistes appartenant au groupe majoritaire (plus nombreux à exposer dans les marchés potiers) ${ }^{11}$. Le processus de création est généralement pris en charge intégralement (façonnage, tournage, modelage, pose de l'émail, cuisson, etc.) y compris parfois la préparation des matières qui les constituent (argiles, émaux, oxydes, etc.) ${ }^{12}$. Leur démarche se caractérise par une forte expérimentation des matières et une recherche constante, ce qui conduit à modifier les dispositifs socio-esthétiques de la céramique.

9 L'exemple de Claude Champy est emblématique de ce groupe de céramistes ${ }^{13}$. Sa posture face à la pratique annonce certaines ruptures avec la "tradition céramique » alors même que les normes du groupe s'y trouvent avec force. Champy soulève la relation ambivalente qui s'établit dans son travail entre la matière et les processus de création :

« l'idée de me servir de l'argile et du feu, pour moi, c'est accepter par avance qu'une partie du résultat final puisse m'échapper (en négatif ou en positif). Je me sens plus au service, à l'écoute de la terre et du feu que le contraire. Je pense qu'il en est en bonne partie de même pour les peintres que j'admire » (Champy in Viatte 2009).

En attribuant à sa pratique une certaine "autonomie ", il se produit en quelque sorte un renversement des rôles : l'artiste est au service de l'art qu'il est en train de faire et non l'inverse. À cette croyance s'ajoute la difficulté technique comme catalyseur de la créativité :

« certaines terres difficiles à travailler, certains fours qui ne fonctionnent pas trop bien ont inventé des céramistes, des non-savoir bien faire ont certainement plus fait pour ouvrir de nouveaux horizons de réflexions et de créations ». 
11 Les principes fondamentaux de la céramique se retrouvent ici dotés d'un pouvoir symbolique face auquel l'artiste voue un respect et se place avec modestie à son écoute :

«J'aime tellement l'odeur de la terre lorsque je rentre dans l'atelier, la vision par les fenêtres des stères de bois rangés dans la cour, attendant patiemment depuis plusieurs années que les choses s'enchaînent naturellement, plus ou moins toutes seules ».

12 Le discours d'indépendance accordé à la matière va jusqu'à considérer l'accident comme une expression de la création (ce qui, auparavant, avait déjà opéré comme un élément artificateur). L'accident s'institue dans une valorisation de l'imprévisible :

« les fentes, craquelures, retraits, surcuissons... tout peut ou devrait engendrer une expression, ce qui au départ n'était qu'un processus s'est transformé en signe pictural. La terre, le feu, j'essaie de leur faire sentir que je les aime, que je les respecte, les admire ».

13 Cette dialectique d'une technique forte, mais à la fois autonome se trouve ancrée entre la maîtrise et l'accident. Si le travail de la matière est présenté comme étant essentiel dans les discours de ces céramistes, l'expérimentation, voire l'invention des outils et instruments pour la création, dans une certaine forme de bricologie ${ }^{14}$, apparaît également comme une caractéristique du groupe. En effet, savoir fabriquer ses propres instruments et équipements (fours, tours, outils, etc.) signifie que l'artiste maîtrise les codes du métier, tout en valorisant l'innovation technique et esthétique. C'est dans cette ambivalence que les « artistes céramistes » jouissent d'un statut intermédiaire les plaçant entre deux mondes, sans en être exclus, ni entièrement intégrés. La maîtrise des techniques de la céramique se pose comme une constante incontournable qui précède la consécration par ses pairs. En revanche, la distanciation de ces mêmes déterminations semble s'imposer comme une condition pour une reconnaissance par les mondes de l'art.

\section{Liberté et attachement dans la technique}

À ce groupe où la dimension technique occupe une place importante s'ajoute l'émergence d'une génération d'artistes plasticiens intéressés par l'univers de la céramique. Ce nouveau pôle est marqué par une forte tendance à la transdisciplinarité ainsi qu'une liberté technique. Les productions céramiques d'artistes pluridisciplinaires et ayant intégré la terre à leur création, tels que Johan Creten, Elmar Trenkwalder, Lucio Fontana, Miquel Barcelo, Jessica Harrison, Zelie Rouby, illustrent ce tendance La notion de liberté n'écarte pas la dimension technique des œuvres, en revanche celle-ci cesse d'être le support principal de l'œuvre. Elle laisse place à une démarche artistique régulièrement associée à l'art contemporain. La technique devient donc le support d'une liberté "et un moyen tendant à une fin ». La médiatisation de ce groupe est assurée par des revues spécialisées en art contemporain telles que Artpress, Art actuel, Art tension, etc., et leurs œuvres font partie des collections publiques et privées d'art contemporain. Les mutations esthétiques de la production céramique à technique libre impliquent les conventions du monde de l'art contemporain.

14 L'artiste belge Johan Creten incarne ce deuxième groupe ${ }^{15}$ par une multiplicité de facteurs: la transdisciplinarité (nombre de ses œuvres sont réalisées en bronze et autres matériaux), son attachement à la matière, le travail de collaboration avec des artisans techniciens, son usage "libre» et expérimental de certaines techniques 
céramiques comme celle du pastillage qui date $\mathrm{du} \mathrm{XVIII}^{\mathrm{e}}$ siècle, et un langage issu de l'univers de l'art contemporain, où la démarche, le concept, l'idée occupent une place prépondérante. Lorsqu'il explique son choix de travailler la céramique comme médium privilégié, Creten évoque une relation avec la matière qui relève d'une expérience singulière et passe par l'expression d'un lien intime tissé naturellement avec les propriétés spécifiques de la matière et qu'il n'aurait pas trouvée dans d'autres médiums :

«Lorsque j'ai touché la terre, j'ai immédiatement senti qu'il y avait une place à prendre. Une place dans l'art, mais aussi une place dans la création (...) Elle permet d'amener de la couleur, des textures, des transparences et surtout une peau qui est très différente de toutes les peaux apportées par d'autres matériaux " (Creten 2013) ${ }^{16}$.

Cette citation communique non seulement le rapport de l'artiste à la matière, mais également l'idée selon laquelle la céramique dans l'art contemporain relèverait d'un combat :

"J'ai commencé à faire des choses que j'incluais dans mes peintures et rapidement, je me suis heurté à une réalité : un artiste qui touche la terre, c'est très tabou dans le milieu de l'art contemporain. Dans les années quatre-vingt, on vous rangeait de côté, "ça ne se faisait pas" ".

Creten est souvent décrit comme un artiste à contre-courant pour s'être intéressé à une pratique délaissée par l'art contemporain. Selon lui, le travail avec une matière perçue comme "sale et humide", se heurte à des interdits et des préjugés qu'il a choisi d'explorer à travers son œuvre dans le but de les dépasser. Les discours de cet artiste relèvent à la fois d'un " engagement "; - son attachement à certaines normes et valeurs de la «communauté céramique» comme la dimension matérielle et technique de l'atelier (il maîtrise les phases de réalisation de ses pièces), le combat pour la reconnaissance de la céramique par la scène artistique en général -, et également d'un univers artistique extérieur - les discours qu'entourent ses œuvres sont teintés d'une rhétorique spécifique à l'art contemporain porté par les propriétés esthétiques, le concept, l'expérience artistique, le sensible, etc.

\section{Le retour de la terre dans l'art contemporain?}

L'intérêt actuel de l'art contemporain pour la céramique et ses institutions se comprend par une multiplicité de facteurs. La revue trimestrielle Artpress 2, intitulée "La céramique au-delà de la céramique ", dont les contributeurs sont pour la plupart des historiens et critiques d'art contemporain affirme dans son introduction, que les espaces de la céramique; les institutions muséales (Cité de la céramique, Sèvres) ${ }^{17}$, certaines initiatives plus modestes et parfois alternatives (Centre de céramique contemporaine de la Borne), les circuits d'enseignement,

« semblent actuellement en pleine expansion. [...] Il y a dix ans à peine, les élèves de

l'École des beaux-arts qui voulaient se former à la céramique peinaient à y trouver un enseignement spécialisé et devaient la plupart du temps se former par euxmêmes. La céramique était encore une technique délaissée, presque ringardisée » ${ }^{18}$.

Les croisements actuels entre céramique et art contemporain soulèvent de nombreuses questions. Par exemple, si la transdisciplinarité en céramique constitue une valeur dans le renouvellement de la pratique, elle est parfois considérée comme étant un risque de faiblesse dans la technique. À l'occasion de la manifestation "Circuits céramiques » en 2010, Frédéric Bodet, qui occupait alors le poste de chargé du département moderne et 
contemporain du musée des Arts décoratifs de Paris, déclarait dans une interview consacrée à la revue numérique ParisArt :

«Je crois que la transdisciplinarité, c'est un facteur de renouveau essentiellement parce que la diffusion de ces œuvres sera plus ouverte. Elle sera faite par des galeries qui ont un pouvoir de conviction et de promotion supérieur. Mais il existe aussi des faiblesses du fait qu'il n'y a pas de spécialisation. Je pense que la céramique est un matériau exigeant, qui ne s'improvise pas. Il y aura toujours des barrières difficiles à franchir, sur des notions de cuisson, d'émail (...). Les plasticiens formés à autre chose ne ressentent pas ça, ou en tout cas ne sont pas capables de le maîtriser. Parfois, il y a une belle intention conceptuelle, mais il y a aussi des déficiences techniques qui limitent un peu l'ampleur de la forme ${ }^{19}$.

Sa posture face à la jeune création céramique met en lumière l'exigence des acteurs du milieu quant à la question de la technique. Il s'agit de «faire ses preuves » tout en ressentant la «force » du matériau. La difficulté technique fonctionnerait donc comme une sélection pour la grandeur artistique du milieu. D'autres acteurs se disent curieux de comprendre les raisons de ce "retour de la terre » dans l'ensemble de la scène artistique contemporaine. François, un collectionneur de céramique, reconnu pour la valeur de sa collection avançait :

« Je plaide pour des études cherchant à déterminer le pourquoi de l'intérêt de plus en plus affirmé de jeunes artistes dans leur rapport avec ce matériau ainsi que vous l'avez compris pour un décloisonnement des comportements et des regards des amateurs $»^{20}$.

Cette idée implique qu'il se serait produit un retour de la terre en même temps que le retour de l'objet et de la matière, dans une époque où l'œuvre ne se trouve plus dans l'objet, mais dans la «dématérialisation, conceptualisation, hybridation, éphémérisation, documentation" (Heinich 2014). Dans son analyse du paradigme de l'art contemporain, Nathalie Heinich souligne que l'extension de l'œuvre au-delà de l'objet traduit l'une des caractéristiques de l'art contemporain. Cette condition se met en pratique à travers "la transgression des frontières où l'expérience des limites". Cependant, l'intérêt grandissant de la céramique dans l'art contemporain soulève des tendances en opposition avec cette "désobjectivation» de l'art contemporain. Cette posture est portée par un sentiment d'attachement à l'objet. Jean, un collectionneur nous confiait qu'il s'est spécialisé dans la céramique suite à son impression de mise à l'écart de l'objet dans l'art contemporain ainsi que par le projet de constituer un ensemble cohérent à travers l'objet :

«J'ai collectionné l'art contemporain. J'ai toujours collectionné... depuis tout jeune.

À un moment donné, j'ai trouvé que l'objet dans l'art contemporain était devenu secondaire. L'objet dans la création plastique était devenu un sous-produit et donc pour les collectionneurs compulsifs... Donc moi qui étais attaché à l'objet, j'ai commencé il y a une quinzaine d'années exclusivement à collectionner la céramique à travers un objet qui est le bol. Mais je ne collectionne pas des bols : je collectionne la céramique, car j'aime avoir une définition encyclopédique, comme un dictionnaire de ma collection. Le bol, non pas pour son utilisation, mais le bol est un objet abstrait qui est un indice de ce que fait le céramiste que je collectionne. C'est un peu la même démarche que la recherche ".

En collectionnant des bols en céramique, Jean pose la question de la fonctionnalité de l'objet. En attribuant le caractère "abstrait ", il écarte une condition problématique de la fonction des objets dans l'art. Pour Thierry Bonnot,

" un objet dit utilitaire a plus souvent plusieurs fonctions distinctes au cours de son existence, successivement ou simultanément ». 
Cette condition devrait inciter les chercheurs à « se méfier des identités fonctionnelles figées devenues gages d'authenticité ». En effet, la double fonctionnalité des objets originelle et attribuée - correspond à des modes d'appropriation qui font :

«partie intégrante de l'histoire des objets et leurs usagers (...) des dimensions techniques, mais aussi historiques et sociales de l'attachement des hommes aux choses ".

L'analyse des situations dans lesquelles les objets se confrontent à la construction de sens par les collectionneurs, montre que le rapport singulier avec l'objet est déterminé par la présence matérielle dans une situation au gré de l'attachement, même si :

« la relation entre sujets et objets ne se limite pas à une relation physique entre le corps du sujet et la matière de l'objet » (Bonnot 2014).

Ainsi, l'attachement des individus aux objets semble être la condition du devenir de l'objet et va déterminer le statut de celui-ci. Bonnot considère que pour étudier les objets matériels, ceux-ci doivent avoir

« une histoire, un devenir fait d'évolutions et de mutations de son statut ; pour cela, il faut qu'il ait à un moment donné de son parcours suscité l'attachement d'un individu, les ressorts de cet attachement étant à la fois collectifs et individuels ».

De son côté, Michelle, collectionneuse de céramique, affirme son besoin de toucher l'objet avant de l'acheter, qu'elle considère fondamental dans la construction de son rapport individuel à l'objet :

«Il y en a qui commencent à mettre "ne pas toucher les pièces". Là on leur fait la tête! Une céramique qu'on peut pas toucher? Si on casse, on paie, d'accord? Mais une céramique c'est important de toucher !»

La condition du toucher est corrélative et nécessaire à la validité de l'objet matériel. Un rapport qui semble s'établir par le contact avec la matière qui le constitue.

Aussi, le retour de la terre dans la création contemporaine s'expliquerait par le désir de certains artistes de retourner "aux sources", au «travail manuel» et à «l'objet». L'expérience de Valentine, une jeune étudiante de la section céramique de l'École d'arts visuels de La Cambre à Bruxelles, illustre ce phénomène. Le projet de Valentine est porté par un attrait pour la matière et les possibilités qu'elle offre :

«J'ai découvert la céramique à un moment donné où j'avais besoin de travailler avec mes mains. (...) Et le travail de la terre permet une infinité de choses. C'est une des rares matières où on peut venir rajouter ou enlever des choses sans avoir besoin d'autre chose que de la terre et de l'eau... c'est très modelable. C'est pas facile, mais c'est assez intuitif ».

Si les céramistes ont pleinement conscience de la difficulté de vivre de leur métier, ils s'engagent dans cette voie dans une quête d'autres formes de reconnaissance, moins portés sur l'aspect financier, mais par l'excellence technique, l'originalité, l'admiration provoquée chez le public et surtout chez leurs pairs. Bajard et Perrenaud relient ce phénomène à la satisfaction du travail manuel des artisans d'art lesquels retireraient

« en outre une satisfaction liée à l'efficacité, la justesse, l'exactitude des techniques et des savoir-faire ainsi que des outils qui sont les supports de leur mise en application. Être un «bon » voire être un "grand » chez ces professionnels, c'est témoigner de sa maîtrise voire de son excellence dans le travail d'un matériau » (Bajard \& Perrenoud 2013).

19 Le choix de Valentine réside également dans le désir de rompre avec les modes de vie « mainstream » de la société actuelle, avec laquelle elle se dit en désaccord, à travers par exemple, l'idée de l'authentique : 
«De manière générale, je pense qu'il y a une envie de retour vers l'authenticité; que ce soit pour acheter ses tomates bios ou pour le fait main... et en France le " Made in France », quête de bonheur trouve son sens dans l'accomplissement d'un projet de vie dont
l'activité artistique est une des composantes. Valentine confiait que le choix d'intégrer
une formation de céramiste, après trois ans d'école d'architecture, avait été très
difficile à accepter par sa famille et son entourage:
« Quand je dis je fais une école de céramique on me demande : et concrètement, tu
vas faire quoi dans la vie? Tu vas vivre de quoi? En fait, ils n'osent pas trop poser
des questions parce qu'ils sont gênés ».

23

mais aussi le principe d'un engagement global et collectif:

«Je pense qu'il y a en ce moment une volonté de revenir aux sources pour essayer d'atténuer tous les désastres qu'on a pu faire en allant vers une consommation de masse et pas du tout contrôlée ».

Le sens donné à l'activité rejoint la mise en œuvre d'une éthique où l'activité n'est pas

« un simple accomplissement individuel, mais où elle remplit une fonction sociale

pour le bien collectif ».(op. cit.) Ainsi, en plus des dispositions sociales et culturelles observées chez ces individus, la
quête de bonheur trouve son sens dans l'accomplissement d'un projet de vie dont l'«intérêt au désintéressement " (Bourdieu 1994) et s'explique par le caractère vocationnel de l'activité, engageant l'individu dans l'exercice d'une passion.

\section{Redessiner les frontières}

Ces nouvelles pratiques redessinent l'espace de la céramique et participent de son décloisonnement. De plus en plus, la création céramique au croisement avec l'art contemporain se lit via le prisme de la déhiérachisation des disciplines, ou d'un mouvement de déspécialisation, voire de détechnicisation (parfois d'anonymisation de la matière). En réponse à ce constat, certains artistes interprètent la pratique en deçà de sa technicité. David Cushway, un artiste du pays de Galles dont l'œuvre (vidéos, installations et performances) mène une réflexion sur les conceptions de l'objet céramique souligne son désaccord face à la distinction entre céramique et art contemporain :

«Pourquoi penser la céramique et l'art contemporain séparément ? La céramique a été longtemps coupable de maintenir un état d'esprit opposant "nous" et "eux". Dans ma pratique artistique, j'ai rencontré des résistances à mon travail par la «fraternité » qui structure le réseau de la céramique [...]. Nous sommes comme le dit Glenn Adamson dans une ère post-industrielle, post-disciplinaire et post-atelier, où les hiérarchies n'existent plus en termes de matériaux et de pratiques ».

Ce témoignage expose la double tension que l'on retrouve fréquemment dans le secteur : d'un côté la négation des hiérarchies et la contestation des distinctions, et de l'autre, les résistances et rejets s'opérant dans le milieu. Lorsque Cushway déclare qu'il a rencontré des résistances à son travail par la « fraternité » céramique, il suggère un changement de perspective. La problématique des clivages est ainsi analysée comme la conséquence d'une auto-marginalisation $\mathrm{du}$ milieu de la céramique. Les hiérarchisations trouveraient leur raison en une posture assumée par le groupe des céramistes qui souhaitent garder une autonomie vis-à-vis des évolutions de l'art. L'article intitulé «La belle céramique est derrière nous » écrit par Thibaut de Ruyter, 
avançait que de plus en plus d'artistes choisissent le support de la céramique pour "repousser (avec humour) les limites du bon goût ». Cet article met en relief la "dichotomie esthétique (et technique) » des jeunes créateurs cassant les codes esthétiques de la «belle céramique ». L'auteur illustre son propos en s'appuyant sur les œuvres d'artistes comme Ehren Tool, Jessica Harrison, Rose Eken, etc. dont le travail puise dans l'imaginaire de la belle céramique pour la détourner et en proposer des sujets déroutants et provocateurs. Il soutient que :

"les artistes contemporains s'affranchissent de la dimension utilitaire, mais aiment à en jouer (...). La force de ces artistes réside dans leur capacité d'expérimentation, quitte à aller à l'encontre du matériau ou à l'utiliser d'une manière très simple » (Ruyter 2013).

En réponse à cet article, Jean François Juillard, un collectionneur de céramique, membre du Club de collectionneurs de céramique, proposait un texte dans lequel il avançait :

" cette belle céramique est loin d'être derrière nous, même si elle est passablement occultée par l'exhibitionnisme contemporain et les tentations de la facilité. La vraie problématique de la céramique actuelle (comme plus généralement de l'art contemporain) ne devrait pas être avant tout le changement, la nouveauté arbitraire, l'effet de choc (...), mais l'approfondissement et le développement de toutes les possibilités du matériau et du métier, la variation continuellement amplificatrice de leurs meilleures composantes ${ }^{21}$.

Ces postures de rejet font écho au discours de rejet de l'art contemporain. Elles sont analysées en sociologie de l'art par Sylvia Girel comme des "formes de réception différentielles » (Girel 2003) ou par Nathalie Heinich comme les réactions du public face aux registres de l'esthétique, de la politique, de la morale, de l'ordre économique, de la valeur du travail, des matériaux utilisés, etc. Les discours recueillis tout au long de l'enquête montrent que les rejets entre les deux pôles de la céramique contemporaine se déploient principalement sur les deux derniers registres. Malgré les critiques émises sur le contenu de ce numéro d'Artpress, celui-ci a été reçu comme un événement important pour l'ensemble du secteur :

"C'est déjà bien qu'ils aient choisi de parler de céramique dans une revue de référence. Même s'il y a des choses très intéressantes, une partie de son contenu est très contestable ${ }^{22}$.

Ce dossier met en lumière l'intérêt grandissant pour la céramique et contribue à la médiatisation du milieu.

\section{La céramique dans le marché de l'art}

Le fait que des revues d'art s'intéressent à la céramique actuelle prouve son accession à de nouveaux domaines de reconnaissance. Cependant, sa présence au sein du marché de l'art, demeure limitée. Nous avons évoqué plus haut le faible recours aux intermédiaires par le milieu. Cette situation trahit pourtant quelques frémissements d'évolution en lien avec les mutations les plus récentes. De plus en plus, les cadres de présentation et de représentation des objets se voient traversés par l'action centrale des intermédiaires. Professionnels, galeristes, collectionneurs, conservateurs, critiques, deviennent les agents dans la construction d'une scène artistique. Leur action est notable dans les espaces où se mettent en œuvre les stratégies de fabrication de la notoriété (Lizé et al. 2014) comme les institutions, les expositions, les collections 
privées, les dispositifs de médiation, etc. De nature composite, le projet de faire reconnaître la céramique par la scène artistique s'articule aux processus d'artification. Ce projet se manifeste dans l'engagement et la rétribution de l'expertise d'amateurs, experts, et intermédiaires hétérogènes. Ainsi, les processus d'évaluation appréhendés comme étant le fruit de la coordination entre acteurs pour s'accorder sur les valeurs économiques et symboliques à travers des conventions, dispositifs, institutions, etc., aboutissent à la construction de la notoriété (ibid). Olivier Roueff analyse ces mécanismes comme

«l'opposition entre les sphères spécialisées de production et le monde des profanes qui en sont les destinataires » (Roueff 2014).

Pour lui,

«la valeur n'a pas de fondement naturel, car elle est simplement fixée, au sein d'une sphère de spécialistes, au terme d'un processus collectif d'évaluation ».

En considérant les processus d'évaluation dans une spécialisation collective, Roueff pose l'intermédiation au centre de l'analyse des processus d'autonomisation. Celle-ci déposséderait les créateurs de certaines compétences inhérentes à l'activité d'intermédiation ou au contraire, elle leur imposerait l'intégralité des étapes du processus de diffusion :

«L'autonomisation des champs de production artistique, morale ou politique, produit simultanément une plus grande autonomie du processus de travail artistique (...) et les artistes peuvent se voir, selon le modèle, dépossédés des moyens de production et de valorisation au profit de grands intermédiaires spécialisés dont ils deviennent dépendants, ou se voient dans l'obligation de s'acquitter eux même des tâches d'intermédiation lorsqu'ils n'ont pas même accès aux petits intermédiaires » (ibid).

29 En effet, l'analyse économique de ces dynamiques s'appuie sur la construction de la valeur par des mécanismes du travail artistique fixés par les rapports de force entre les travailleurs et le marché. Les tensions inhérentes aux processus d'accès à la reconnaissance, à la notoriété et à la consécration de l'univers artistique, s'expriment aussi et surtout à travers la contestation des politiques publiques, des logiques de fonctionnement des espaces de diffusion et de commercialisation ${ }^{23}$ :

«Les artistes utilisant la céramique ne sont guère aidés depuis quelques années par les musées ni au niveau des expositions ni au niveau des achats. Les galeries "dédiées à la céramique » ont la plupart peu de moyens et manquent souvent de lisibilité dans leurs lignes de choix. Les galeries « autres » qui montrent des artistes utilisant le médium céramique ne le font qu'avec des artistes la pratiquant occasionnellement ou bien en montrant des réalisations faites par des tiers (Manufacture de Sèvres par exemple) (...) ${ }^{24}$.

30 Ce désaccord avec le milieu renvoie à une conception du marché de la céramique ancrée dans une double marginalité. D'un côté se trouvent le marché et ses instances régulatrices considérées par certains acteurs sociaux comme défavorables, et de l'autre, une communauté professionnelle construite autour de normes et éthiques perçues comme des « chapelles ».

La typologie de galeries d'art contemporain proposée dans l'ouvrage de N. Moreau, D. Sagot-Duvauroux et recensé par Sylvia Girel (Benhamou et al. 2001) distingue les galeries de négoce et celles de promotion en quatre sous-groupes :

- Les galeries qui défendent un art qualifié par elles de figuratif, peu présentes sur les foires, elles évoluent en marge du marché international. 
- Les «jeunes petites» galeries fortement impliquées dans l'avant-garde internationale, soutenues par les pouvoirs publics par le biais d'achat.

- Les galeries aux mêmes engagements esthétiques que les précédentes, mais plus anciennes et donc reconnues et présentes à l'échelle internationale, employant un ou plusieurs salariés.

- Les galeries les plus anciennes qui se partagent entre la promotion d'artiste et un travail de marchand.

31 Si l'on rapproche ce modèle générique à la céramique à technique forte, celle-ci serait surtout représentée par le premier et le dernier groupe. C'est-à-dire qu'elle reste promue par des petites galeries spécialisées, mais reconnues au sein du milieu de la céramique. Le travail de l'intermédiation est aussi assuré par le céramiste lui-même. Nombre d'entre eux commercialisent leurs productions, organisent des festivals, symposiums, expositions, écrivent et publient des livres, etc. Ils bénéficient d'une certaine reconnaissance institutionnelle, mais celle-ci reste spécialisée dans le milieu. La céramique à technique forte est médiatisée à travers une presse spécialisée (la Revue de la céramique et du verre) et de l'ensemble du secteur des métiers d'art (Ateliers d'Art de France). Elle retrouve a contrario, une faible reconnaissance au sein de la scène de l'art contemporain.

À l'inverse, la céramique à technique libre a nettement plus recours aux intermédiaires que ses collègues céramistes de l'autre groupe. Elle rencontrerait une plus forte reconnaissance au sein des deuxièmes et troisièmes groupes de galeries proposés par N. Moreau, D. Sagot-Duvauroux. Elle est représentée sur la scène internationale par le biais de contrats d'exclusivité artistique.

Les paramètres de reconnaissance sont également identifiables selon la valeur commerciale attribuée aux objets dans le circuit marchand de l'art. Les sites de ventes aux enchères sur internet, par exemple, rendent compte des écarts existants dans l'estimation de la valeur des objets d'après leur appartenance à l'un ou l'autre pôle de la céramique. À titre d'exemple, une "pièce" de Claude Champy, est estimée à 1800 euros ${ }^{25}$ tandis qu'une "œuvre» de Johan Creten, représenté par la galerie Perrotin, est estimée à plus de dix fois ce prix, soit 20000 euros ${ }^{26}$. Les échelles de légitimité en matière de céramique contemporaine sont également perceptibles à travers la terminologie couramment attribuée aux objets. L'usage de la notion de pièce et d'œuvre est alterné selon les conventions de l'espace qui l'emploie. Le terme "pièce » est répandu dans le milieu de la céramique et il est usuel de parler d' «œuvre " pour désigner l'objet produit par un artiste, que ce soit en céramique ou dans un autre matériau.

L'observation du monde social de la céramique actuelle a montré qu'il s'y opère une articulation entre les pratiques majoritaires liées à une identité professionnelle inscrite entre l'art et le métier, et d'autres pratiques minoritaires plutôt portées sur l'art contemporain. Ces croisements cristallisent une forte transformation des pratiques, des objets, des dispositifs esthétiques et techniques, etc. En nous intéressant aux discours des acteurs impliqués dans le milieu, nous avons tenté de mieux comprendre comment opèrent ces processus. En effet, les témoignages des collectionneurs, conservateurs, artistes, etc., mettent en lumière les mécanismes de l'engagement et de l'attachement artistiques, mais aussi les accords et les tensions internes face aux changements récents. De ces discours se dégagent des postures guidées par un système de représentations qui dévoilent des relations symboliques et matérielles, à la pratique et 
aux objets parfois contradictoires. Bien que la céramique existe à travers l'idée d'une communauté artistique autonome, elle existe aussi et surtout dans une hétérogénéité de pratiques. Face à cette diversité, les acteurs du milieu mettent en œuvre des stratégies, discutent les limites et les partages, et se positionnent par des engagements individuels et collectifs pour la reconnaissance artistique du milieu: si bien que l'espace artistique dans son ensemble bénéficie d'un engouement de plus en plus grandissant, sa reconnaissance semble davantage souhaitée qu'effective. En effet, la céramique reste un champ artistique méconnu, plus que rejeté et ses acteurs peinent à trouver des consensus entre les discours, la terminologie, les horizons d'attente, les dispositifs de médiation, etc.

Le constat d'une polarisation au sein même des pratiques minoritaires de la céramique contemporaine découle d'un questionnement d'ordre épistémologique, révélateur d'une reconnaissance différenciée au sein des mondes de l'art. La diversité de discours a montré que la notion de "technique» joue un rôle déterminant au moment d'interroger les pratiques et les réalités du milieu. Le traitement différentiel de la technique, face à la mise en scène d'une culture céramique en contact avec l'art contemporain, renouvelle l'espace artistique, ainsi que les normes qui le composent. C'est cette hypothèse, liée à la réflexivité de la technique dans l'objet, que l'étude a testée en tentant de vérifier si les représentations et la place de la technique céramique chez les publics experts coïncidaient avec la polarisation instituée par les acteurs des mondes de l'art.

\section{BIBLIOGRAPHIE}

Bajard, F. 2014 Sociologie des céramistes d'art en France. L'invention d'un groupe socio-professionnel: pratiques et manières d'être. Thèse de doctorat (en cours de publication) Thèse. Université de Lausanne, Institut des Sciences Sociales.

-2012 « Du travail d'atelier au collectif : l'inscription professionnelle ambivalente des “céramistes créateurs" ». Sociologie de l'Art 21(3) : 43-64.

Bajard, F., Perrenoud, M. 2013 « Ça n'a pas de prix ». Diversité des modes de rétribution du travail des artisans d'art. Sociétés Contemporaines 91(3) : 93.

Benhamou, F., Moureau, N., Sagot-Duvauroux, D. 2001 Les Galeries d'art contemporain en France : portrait et enjeux dans un marché mondialisé. Paris : La Documentation française : Ministère de la culture et de la communication, département des études et de la prospective.

Bonnot, T. 2014 L'Attachement aux choses. Paris : CNRS Éditions.

Bourdieu, P. 1994 « Un acte désinteressé est-il possible? » in Raisons pratiques. Paris : Seuil : 149-167.

Brunet, M., Giacomotti, J., Pecker, A. 2015 Céramique. Encyclopædia Universalis (en ligne). http:// www.universalis.fr/encyclopedie/ceramique/ 
Davallon, J. 2006 Le Don du patrimoine : une approche communicationnelle de la patrimonialisation. Paris : Hermès Science-Lavoisier.

- 2000 L'Exposition à l'œuvre : stratégies de communication et médiation symbolique. Paris :

L'Harmattan.

Girel, S. 2003 La Scène artistique marseillaise des années quatre-vingt-dix. Une sociologie des arts visuels contemporains. Paris : L'Harmattan.

Heinich, N. 2014 Le Paradigme de l'art contemporain : structures d'une révolution artistique. Paris : Gallimard.

- 2005 L'Élite artiste : excellence et singularité en régime démocratique. Paris : Gallimard.

Heinich, N., Shapiro, R. 2012 De l'artification : enquêtes sur le passage à l'art. Paris : Éditions de l'École des hautes études en sciences sociales.

Jourdain, A. 2012 « Réconcilier l'art et l'artisanat : Une étude de l'artisanat d'art ». Sociologie de l'Art 21(3) : 19.

Lahire, B. 2012 Monde pluriel : penser l'unité des sciences sociales. Paris : Éditions du Seuil, DL 2012.

Lizé, W., Naudier, D., Sofio, S. 2014 Les Stratèges de la notoriété : Intermédiaires et consécration dans les univers artistiques. Paris : Éditions des archives contemporaines.

Olivier de Sardan, J.-P.. 1995 « La Politique du terrain ». Enquête : 71-109.

Roueff, 0. 2014 «Les Mécanismes de valorisation à l'épreuve des systèmes d'intermédiation » in Les Stratèges de la notoriété: Intermédiaires et consécration dans les univers artistiques. Paris : Éditions des archives contemporaines : 185-202.

Ruyter, T. de 2013 « La Belle céramique est derrière nous ». Artpress 2. La céramique au-delà de la céramique (31) : 40.

Stoichita, Victor A. 2011 « Entre prouesse et dérision ». Ateliers d'anthropologie [En ligne] (35) consulté le 26 mars 2016. URL : http://ateliers.revues.org/8793 ; DOI : 10.4000/ateliers.8793.

Stoichita, Victor A., Grimaud, E., Jones, G. 2011 « Préambule », Ateliers d'anthropologie [En ligne] (35), consulté le 26 mars 2016. URL : http://ateliers.revues.org/8838; DOI : 10.4000/ateliers.8838 : $\S 6$.

Viatte, G. 20098 artistes \& la terre [Claude Champy, Bernard Dejonghe, Philippe Godderidge, Jacqueline Lerat, Michel Muraour, Setsuko Nagasawa, Daniel Pontoreau et Camille Virot]. Banon : Éditions ARgile.

\section{NOTES}

1. Il existe en céramique des techniques et des processus très divers (raku, céladon, cristallisation, terre vernissée, etc.), de nombreux matériaux et argiles (chamotte, porcelaine, rouge, verte, grés, etc.), équipements (fours à gaz, à bois ou électrique), systèmes de cuisson, manufactures (modelage à la main, au tour, moulage, etc.).

2. La notion d'amateur de céramique désigne dans ce travail l'attachement et l'amour pour la création céramique et non pas le pratiquant non professionnel de céramique.

3. Ateliers d'Art de France est né en 1868 en tant que chambre syndicale des métiers d'art à l'initiative d'un groupe de céramistes et constitue aujourd'hui la principale organisation professionnelle des métiers d'art en France. Il compte 6000 professionnels (artisans, artistes, manufactures d'art, etc.) et cinq espaces d'exposition et vente dans toute la France (dont la Galerie Collection). 
4. Les modes de participation aux mondes de l'art déterminent différents types d'acteurs dont Becker dresse une typologie. Les professionnels intégrés sont ceux qui détiennent le savoir-faire technique, les aptitudes sociales et le bagage intellectuel pour la réalisation d'œuvres d'art en accord avec l'activité. Les francs-tireurs sont ceux qui apportent des innovations que le monde de l'art n'accepte pas immédiatement. Ils vont se heurter à des résistances de la part des autres membres puisqu'ils transgressent les conventions, bien qu'ils respectent certaines d'entre-elles. Toutefois, les innovations pourront être assimilées par les professionnels intégrés avec le temps et la configuration des mondes de l'art.

5. La porcelaine est une matière dont la production est généralement associée aux services de table d'une élite bourgeoise.

6. Le Prix Tourner (Turner Prize en anglais) est un prix d'art créé en 1984 pour célébrer le développement de l'art contemporain. Il est décerné chaque année à « un artiste britannique de moins de cinquante ans pour une exposition exceptionnelle ou une autre présentation de son travail au cours des douze mois précédents. URL : http://www.tate.org.uk. Consulté le 7 octobre 2014.

7. Depuis ses débuts la manifestation visait à accompagner la création en céramique, mais c'est en 2005 que la ville annonce une biennale sur le thème du corps, l'atelier et le paysage, avec un soutien financier plus important, de nombreux partenariats (dont le Musée de Sèvres), et une ouverture vers l'international. La XVIII édition de la biennale a eu lieu en 2015 et s'intitulait «Objectif terre ».

8. "Circuits céramiques, en septembre 2010, Paris se transforme en écrin céramique ", Site web de la Cité de la céramique, Sèvres, mis en ligne en 2010. URL: http://www.sevresciteceramique.fr/ site.php?type=P\&id=297. Consulté le 12 octobre 2014.

9. Le choix de situer la frontière sur les plans technique et esthétique vise également à appréhender le partage au delà des catégories traditionnelles, tantôt chargées, tantôt controversées, telles que «céramique traditionnelle » versus «céramique contemporaine » ou « innovante », « savante », etc.

10. Fondée en 1952, l'Académie internationale de la céramique est affiliée à l'Unesco à titre consultatif et son siège officiel se situe au musée Arianna, musée de céramique et du verre de Genève. La composition actuelle de l'Académie internationale de la céramique est de 584 membres issus de 51 pays du monde.

11. Certains marchés ont acquis une notoriété certaine comme ceux des Tupiniers à Lyon, de Saint Quentin-la-poterie, le marché biennale Argilla, Aubagne, etc. Ces marchés opèrent une importante sélection des exposants par l'expertise d'un comité constitué de professionnels, notamment. La participation aux Journées de la céramique de Saint Sulpice n'est pas incompatible avec l'exposition au sein d'une galerie parisienne. Cependant, la participation fréquente d'un céramiste dans un marché céramique rend difficile l'accès à d'autres espaces de promotion et de commercialisation qui lui sont mieux consacrés tels que les galeries.

12. Certains vont jusqu'à fabriquer certains équipements liés aux processus techniques : un four (à bois, à gaz, etc.), un tour et différents outils associés.

13. En 2010, le musée de la Cité de la céramique, Sèvres lui consacrait une exposition rétrospective intitulée « Terre complice ».

14. Le néologisme «bricologie » est défini sur le site de la Villa Arson comme une étude de la culture technique dans les arts : «L'étude des manières de faire exister une œuvre d'art, qu'elles relèvent $\mathrm{du}$ bricolage manuel, du savoir-faire artisanal, de la production industrielle, de la délégation, ou de l'élaboration d'une chaîne opératoire qui mélange ces différentes possibilités ". URL : http://www.villa-arson.org/2014/02/bricologie/. Consulté le 26 mars 2016.

15. Il a effectué durant sa carrière de nombreuses résidences d'artiste dans le monde entier (Villa Arson, Nice (1993), Villa Medici, Rome (1996), Cité Céramique, Sèvres (de 2004 à 2007), etc. Il 
réside et travaille en France depuis 1991. Il est représenté par la galerie d'art contemporain Perrotin.

16. Entretien de Johan Creten par Léa Chauvel-Lévy pour le magazine numérique d'art, Slash. URL : slash-paris.com/en/articles/johan-creten-interview - février 2013 - Consulté le 26 mars 2016.

17. Les nouvelles orientations de la Cité de la céramique, Sèvres, se situent d'emblée dans un mouvement de renouveau. La céramique contemporaine est présente dans ce lieu (créé en 1824) à travers une promesse institutionnelle structurée autour de la spécificité patrimoniale et technique avec une ouverture vers la création actuelle. Cela est visible par différents aspects tels que l'inauguration du parcours de céramiques contemporaines en mai 2015, un programme de résidences artistiques où designers et artistes de tous horizons sont invités à explorer le matériau, en s'employant aux techniques de Sèvres et en apportant un regard nouveau, via l'organisation et la participation à de nombreuses manifestations en lien avec l'art contemporain, etc. La Cité de la céramique se place également dans une perspective de restructuration des modèles institutionnels et académiques grâce à des positionnements stratégiques en relation avec les réalités politiques et économiques des mondes de l'art et un marché exigeant en termes d'innovation, dont les objectifs de performativité sont réels (la Cité de la céramique, Sèvres est devenue Établissement public depuis 2010).

18. «La céramique au delà de la céramique ", Artpress 2, n 31, novembre-décembre 2013, janvier 2014.

19. Entretien avec Frédéric Bodet, conservateur du département de la céramique contemporaine à la Cité de la céramique, Sèvres, Enquête décembre 2013.

20. Propos d'un amateur-collectionneur, membre du Club de collectionneurs de céramique, ayant répondu au questionnaire en ligne : «À la rencontre des publics de la céramique » 2014.

21. «Réactions au numéro d'Artpress 2 consacré à la céramique", Site web du club de

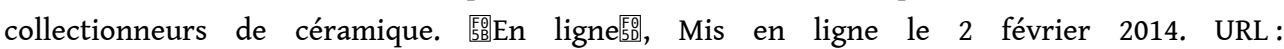
lesceramophiles.org/actu/artpresse-consacre-un-numero-special-a-la-ceramique.html. Consulté le 26 mars 2016.

22. Propos d'un amateur-collectionneur, membre du Club de collectionneurs de céramique, Enquête 2014.

23. Depuis les trente dernières années, de nombreuses galeries spécialisées ont vu le jour, mais aussi nombreuses sont celles qui ont fermé leurs portes.

24. Propos d'un amateur-collectionneur, membre du Club de collectionneurs de céramique, ayant répondu au questionnaire en ligne : « À la rencontre des publics de la céramique », Enquête 2014. 25. Information issue du site web de vente d'œuvres d'art Artactif. URL : artactif.com/champyclaude/galerie/alchimie_20595.htm\#.VdIum14i3Sg. Consulté le 26 mars 2016.

26. Information issue du site web de ventes aux enchères d'objets d'art Artprice. URL: http:// fr.artprice.com/artiste/186571/johan-creten. Consulté le 26 mars 2016.

\section{RÉSUMÉS}

En partant de l'analyse d'entretiens réalisés auprès de divers acteurs du monde de la céramique (professionnels, collectionneurs, conservateurs, etc.), l'article examine le retour de la céramique au sein de l'espace artistique contemporain. Il rend compte du projet de reconnaissance porté par la communauté de la céramique, de plus en plus investi par un groupe d'artistes 
contemporains et dont la pratique se positionne dans une dynamique de décloisonnement. Les transformations récentes du milieu - où se conjuguent technique, "savoir-faire » et langages contemporains - nous renseignent non seulement sur le basculement qui s'opère au sein de la pratique, mais aussi dans un système artistique polarisé par les représentations esthétiques et techniques de la céramique.

Based on the analysis of interviews done with various actors of the world of ceramics (professionals, collectors, curators, etc.) this article investigates the return of ceramics into the sphere of contemporary art. It tackles the project conducted by the community, increasingly invested by contemporary artists whose practices attempt to break boundaries. The recent changes in the field - where technical skills, "know-how" and contemporary trends are tightly combined - indicates that, not only a shift is operating in the practices, but also in the artistic system, polarized by aesthetics and technical representations of ceramics.

INDEX

Keywords : ceramics, contemporary arts, crafts, technical skills, mediation, representations

Mots-clés : céramique, art contemporain, artisanat d'art, technique, médiation, représentations

\section{AUTEUR}

\section{ELISA ULLAURI LLORÉ}

Université d'Avignon, Centre Norbert Elias et Aix-Marseille Université, Lames 